Mohamed Hassanein, MD

Department of Nephrology and Hypertension,

Glickman Urological and Kidney Institute,

Cleveland Clinic
George Thomas, MD

Department of Nephrology and Hypertension, Glickman Urological and Kidney Institute, Cleveland Clinic
Jonathan Taliercio, DO

Department of Nephrology and Hypertension,

Glickman Urological and Kidney Institute,

Cleveland Clinic

\title{
Management of acute kidney injury in COVID-19
}

\section{Posted May 12, 2020}

\section{ABSTRACT}

Acute kidney injury has been reported in as many as $29 \%$ of COVID-19 patients. Reported risk factors include elevated baseline serum creatinine, elevated blood urea nitrogen, acute kidney injury, proteinuria, and hematuria. Suspected causes include sepsis and acute tubular necrosis resulting from renal hypoperfusion, cytokine release syndrome, direct viral invasion, renal medullary hypoxia secondary to alveolar damage, rhabdomyolysis, and cardiorenal syndrome due to viral myocarditis.

\section{INCIDENCE OF AKI}

Acute kidney injury (AKI) has been reported to occur in $3 \%$ to $29 \%$ of COVID-19 patients. ${ }^{1-5}$ The wide range of reported incidence is likely due to inconsistent use of AKI definitions and to variable incidence in people hospitalized with mild symptoms vs critically ill patients. Published reports indicate that kidney replacement therapy will be needed in about 3\% to $17 \%$ of COVID-19 patients who develop AKI. 4,5 Patients with AKI have a higher risk of mortality, and predictors for mortality in a study of 701 COVID-19 patients included elevated baseline creatinine, blood urea nitrogen, AKI, proteinuria, and hematuria. ${ }^{1}$

\section{ETIOLOGY OF AKI}

The pathogenesis of AKI in COVID-19 is unclear due to the limited availability of kidney biopsy reports. It is intriguing that SARS-CoV-2 utilizes angiotensin converting enzyme 2 as the receptor for entry, which is highly expressed in the proximal tubules in the kidney. ${ }^{6}$ Suspected etiologies for AKI include sepsis and acute tubular necrosis resulting from renal hypoperfusion, cytokine release syndrome, direct viral invasion, renal medullary hypoxia secondary to alveolar dam-

The statements and opinions expressed in COVID-19 Curbside Consults are based on experience and the available literature as of the date posted. While we try to regularly update this content, any offered recommendations cannot be substituted for the clinical judgment of clinicians caring for individual patients.

doi:10.3949/ccjm.87a.ccc034 age, rhabdomyolysis, and cardiorenal syndrome due to viral myocarditis. ${ }^{7-9}$ A postmortem analysis of 26 patients in China showed evidence of acute tubular necrosis and, less commonly, white blood cell casts and bacteria suggesting pyelonephritis. Viral particles were also identified within the podocytes and proximal tubular cells, supporting direct viral invasion as a possible mechanism for AKI. Additionally, patients with elevated serum creatinine phosphokinase had pigmented casts on autopsy, suggesting rhabdomyolysis as a possible cause. Interestingly, peritubular and glomerular capillary luminal obstruction by erythrocyte aggregation in some patients also suggests a possible role of hypercoagulability. ${ }^{8}$ Proteinuria and hematuria have been reported in some patients, although it is unclear whether this is due to underlying chronic kidney disease or to infection-mediated glomerulonephritis ${ }^{1}$ : there is a report of collapsing glomerulopathy on kidney biopsy in an African American patient with nephrotic-range proteinuria and underlying high-risk APOL1 genetic variant. ${ }^{10}$

\section{EVALUATION OF AKI}

The evaluation of AKI in COVID-19 patients should follow the general approach to workup and diagnosis of AKI, utilizing the broad framework of prerenal, intrarenal, and postrenal causes. A careful history and physical examination, hemodynamic and volume assessment, and review of possible nephrotoxic medications should be undertaken. Urinalysis with sediment examination is helpful to differentiate the causes of AKI. A positive urine dipstick for hemoglobin with no erythrocytes on microscopy could signify rhabdomyolysis. Muddy brown casts and white blood cell casts on urine sediment examination would suggest acute tubular necrosis and acute interstitial nephritis or pyelonephritis, respectively. Measurement of urine sodium may help differentiate prerenal causes from acute tubular necrosis. Kidney ultrasonography can help identify underlying medical renal disease and rule out obstruction. Kidney biopsy should be considered 
in patients in whom the etiology of AKI is unclear, and if the benefits of identifying a cause outweigh the risks of performing the procedure. ${ }^{11}$

\section{TREATMENT OF AKI}

Supportive management includes optimizing hemodynamic and volume management and discontinuation of nephrotoxic medications. The indications and timing of dialysis initiation remains the same for COVID-19 patients as for other AKI patients. Additional therapies undergoing active investigation that are not limited to renal disease include antiviral and anti-inflammatory therapy, such as remdesivir, lopinavir-ritonavir, and tocilizumab. ${ }^{7-12}$ The efficacy and safety of these drugs have not been reported. Although there is no evidence to support acute nephrotixicty with these drugs, some renal adverse effects have been reported. Protease inhibitors should be used cautiously in kidney transplant recipients as they can increase the blood levels of immunosuppressive medications (including the calcineurin inhibitor tacrolimus), which can worsen AKI. ${ }^{13}$ Chloroquine and hydroxychloroquine have been rarely associated with renal podocytopathy mimicking Fabry disease. ${ }^{14,15}$ Adalimumab, a monoclonal antibody against tissue necrosis factor alpha, is associated with autoimmune glomerulonephritis. ${ }^{15}$ Bacterial superinfection necessitates initiation of antibacterial therapy, which may also have nephrotoxic effects. ${ }^{11}$

Continuous kidney replacement therapy is the preferred modality of dialysis in patients with hypotension. Theoretically, convective forms of kidney replacement therapy (hemofiltration) have been suggested to improve the removal of cytokines, but they have not been proven to have better outcomes than diffusive forms (hemodialysis). There is increased risk of clotting of hemodialysis filters due to cytokine-induced hypercoagulability, so clinicians should attempt to anticoagulate all patients that are on dialysis if there is no contraindication. ${ }^{16}$ Hemoperfusion filters, designed to remove bacteria and viruses from the blood, are being studied in trials in COVID19 patients in Germany and Italy. ${ }^{17}$ Additionally, extracorporeal membrane oxygenation (ECMO) may help improve kidney oxygenation and reduce the risk of medullary hypoxia and both kidney replacement therapy and extracorporeal membrane oxygenation have been used in conjunction with one another. ${ }^{9}$

\section{PRESERVING RESOURCES}

Due to the high rate of AKI requiring kidney replacement therapy in critically ill patients, a careful daily assessment of available resources is needed: a dialysis dashboard to track equipment, supplies, personnel, and patients should be implemented. Strategies to limit frequent patient contact include extension tubing that allows dialysis machines to be placed outside the patient rooms, and the use of bedside video monitors. Due to disruptions in the supply chain, healthcare systems may experience difficulty with availability of supplies including dialysis filters, tubing, and premade replacement fluid. Some institutions have switched from hemofiltration to hemodialysis and are producing dialysis solutions in-house. ${ }^{16}$ The Cleveland Clinic now has an online resource at www.youtube. $\mathrm{com} /$ watch $\mathrm{v}=1 \mathrm{ektoZGu} 83 \mathrm{M} \&$ feature $=\mathrm{emb}$ logo, which is a step-by-step tutorial for in-house dialysis fluid production. Others have suggested urgentstart peritoneal dialysis for patients with AKI when resources are limited. ${ }^{16}$

\section{SUMMARY}

Patients with COVID-19 have an increased risk of AKI and death. The etiologies of AKI are multifactorial, and data from larger case series are needed. Management of AKI is supportive, and extracorporeal therapies may be required in critically ill patients. Healthcare systems should closely track dialysis resources, and best-care practices should be shared to optimize care in settings of limited resources.

\section{REFERENCES}

1. Cheng $\mathbf{Y}$, Luo $\mathbf{R}$, Wang $\mathbf{K}$, et al. Kidney disease is associated with in-hospital death of patients with COVID-19. Kidney Int. 2020. doi:10.1016/j.kint.2020.03.005

2. Guan W, Ni Z, Hu Y, et al. Clinical Characteristics of Coronavirus Disease 2019 in China. N Engl J Med. February 2020:NEJMoa2002032. doi:10.1056/NEJMoa2002032

3. Chen N, Zhou M, Dong X, et al. Epidemiological and clinical characteristics of 99 cases of 2019 novel coronavirus pneumonia in Wuhan, China: a descriptive study. Lancet. 2020. doi:10.1016/S0140-6736(20)30211-7

4. Yang $\mathbf{X}$, Yu Y, Xu J, et al. Clinical course and outcomes of critically ill patients with SARS-CoV-2 pneumonia in Wuhan, China: a singlecentered, retrospective, observational study. Lancet Respir Med. February 2020. doi:10.1016/S2213-2600(20)30079-5

5. Richardson S, Hirsch JS, Narasimhan M, et al. Presenting Characteristics, Comorbidities, and Outcomes Among 5700 Patients Hospitalized With COVID-19 in the New York City Area. JAMA. April 2020. doi:10.1001/jama.2020.6775

6. Hoffmann M, Kleine-Weber H, Schroeder S, et al. SARS-CoV-2 Cell Entry Depends on ACE2 and TMPRSS2 and Is Blocked by a Clinically Proven Protease Inhibitor. Cell. 2020;181(2):271-280.e8. doi:10.1016/j.cell.2020.02.052

7. Naicker S, Yang CW, Hwang SJ, Liu BC, Chen JH, Jha V. The Novel Coronavirus 2019 epidemic and kidneys. Kidney Int. 2020. doi:10.1016/j.kint.2020.03.001

8. Hua Su, Ming Yang, Cheng Qan, Li-Xia Yi, Fang Tang, Hong-Yan Zhu, Fan Yi, Hai-Chun Yang, Agnes B Fogo XN and CZ. Renal histopathological analysis of 26 postmortem findings of patients with COVID-19 in China. Kidney Int. 2020;Article in.

9. Ronco C, Reis T. Kidney involvement in COVID-19 and rationale for extracorporeal therapies. Nat Rev Nephrol. April 2020. doi:10.1038/ s41581-020-0284-7 
10. Kissling S, Rotman S, Gerber C, et al. Collapsing glomerulopathy in a COVID-19 patient. Kidney Int. April 2020. doi:10.1016/j. kint.2020.04.006

11. Moore PK, Hsu RK, Liu KD. Management of Acute Kidney Injury: Core Curriculum 2018. Am J Kidney Dis. 2018. doi:10.1053/j. ajkd.2017.11.021

12. Sanders JM, Monogue ML, Jodlowski TZ, Cutrell JB. Pharmacologic Treatments for Coronavirus Disease 2019 (COVID-19). JAMA. April 2020. doi:10.1001/jama.2020.6019

13. Perico L, Benigni A, Remuzzi G. Should COVID-19 Concern Nephrologists? Why and to What Extent? The Emerging Impasse of Angiotensin Blockade. Nephron. 2020. doi:10.1159/000507305

14. Wu S-Z, Liang X, Geng J, Zhang M-B, Xie N, Su X-Y. Hydroxychloro- quine-induced renal phospholipidosis resembling Fabry disease in undifferentiated connective tissue disease: A case report. World J Clin Cases. 2019;7(24):4377-4383. doi:10.12998/wjcc.v7.i24.4377

15. Izzedine H, Jhaveri KD, Perazella MA. COVID-19 therapeutic options for patients with kidney disease. Kidney Int. March 2020. doi:10.1016/j.kint.2020.03.015

16. Burgner A, Ikizler TA, Dwyer JP. COVID-19 and the Inpatient Dialysis Unit. Clin J Am Soc Nephrol. April 2020:CJN.03750320. doi:10.2215/ CJN.03750320

17. ExThera's Affinity Blood Filter Is Used to Treat COVID-19 Patients. http://www.extheramedical.com/news-releases/2020/3/26/extherasaffinity-blood-filter-is-used-to-treat-covid-19-patients. Published 2020. 